\title{
Mouton, dessine-moi un pays
}

L'élevage ovin et l'identité régionale en Patagonie

Fernando Raul Coronato et Jean-François Tourrand

\section{(2) OpenEdition}

Journals

Édition électronique

URL : https://journals.openedition.org/tc/7475

DOI : $10.4000 /$ tc. 7475

ISBN : 0248-6016

ISSN : 1952-420X

Éditeur

Éditions de l'EHESS

\section{Édition imprimée}

Date de publication : 1 mai 2015

Pagination : 161-181

ISBN : 9782713224874

ISSN : 0248-6016

\section{Référence électronique}

Fernando Raul Coronato et Jean-François Tourrand, « Mouton, dessine-moi un pays », Techniques \& Culture [En ligne], 63 | 2015, mis en ligne le 18 décembre 2018, consulté le 29 septembre 2022. URL : http://journals.openedition.org/tc/7475; DOI : https://doi.org/10.4000/tc.7475 


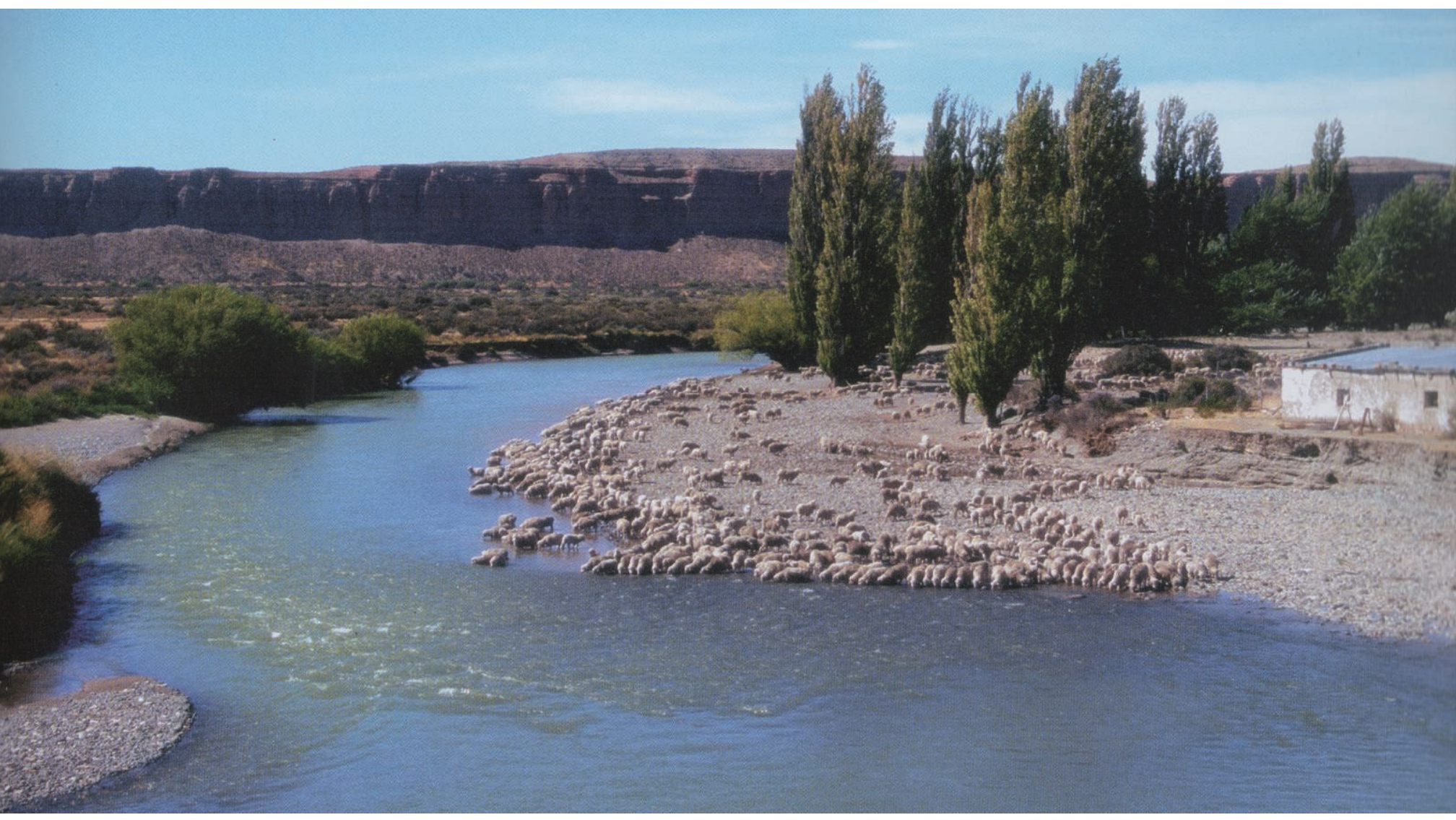

- Jorge Miglioli 


\section{MOUTON, DESSINE-MOI UN PAYS}

\section{L'élevage ovin et l'identité régionale en Patagonie}

Lorsque le Petit Prince, loin de partout, demandait à Antoine de Saint-Exupéry de lui dessiner un mouton, il aurait bien pu se trouver en Patagonie. Sur la route de l'Aéropostale le pilote-écrivain survolait le Sahara aussi bien que la Patagonie, terminus de la ligne, et à cette époque-là, autour de 1930, la Patagonie était encore une terre de pionniers, soient-ils des éleveurs ou des aviateurs. .

Dans cet article nous retraçons les étapes du cycle ovin en Patagonie, responsable d'avoir déclenché l'organisation territoriale et sociale de cette région, et nous analysons les causes de son succès retentissant suivi d'un long déclin agonisant. Nous arguons qu'il est cependant trop tôt pour sonner le glas du mouton en Patagonie en raison du poids de l'élevage ovin dans la définition de l'identité régionale.

Les parcours de Patagonie sont les plus au sud de la planète et parmi les derniers à avoir été mis en exploitation. L'élevage ovin dans la région a débuté il y a un peu plus d’un siècle, et a connu un fort développement pendant près de cinq décennies, jusqu'à la crise

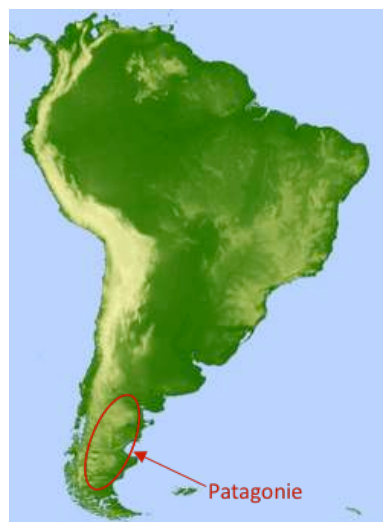
mondiale des années 1930 pour continuer à s'étaler au ralenti dans le reste de la région, qui n’a été pleinement occupée qu'aux années 1950.

L'expansion rapide de l'élevage ovin dans la région est allée de pair avec l'occupation hâtive des terres de parcours de productivités très différentes. L'aubaine lainière représentée par le prix élevé du kg de laine dissimula longtemps la véritable défaillance agronomique du système. En effet, dans cette zone aride et froide où l'exploitation moyenne fait environ 10000 hectares, s'il y avait des parcours qui supportaient des charges de l'ordre d'un ovin à l'hectare, cas des terres les plus arrosées, situées au sud ou le long de la cordillère andine, la plupart des autres parcours ne supportaient que des charges bien inférieures, de l'ordre 
de deux à trois têtes pour une dizaine d'hectares. Ils ont ainsi été fortement dégradés en moins de quelques décennies pour n'être aujourd'hui que des aires désertiques. Par ailleurs, le développement de l'élevage ovin en Patagonie à partir des années 1880 n'était pas un phénomène isolé mais il s'insérait bien dans le contexte national argentin d'expansion des frontières politiques aussi bien que dans le contexte international d'élargissement des frontières agricoles dans des zones tempérées.

Ainsi, le boom ovin en Patagonie, avec comme point de départ les îles Malouines au sud et la Pampa au nord, concerna aussi bien le secteur argentin de Patagonie que le chilien, les deux constituants une unité socio-économique fédérée autour du mouton. Une région pratiquement autarcique s'est organisée à partir de Punta Arenas, sur le détroit de Magellan, et produisit en 1914 à elle seule un tiers de toute la laine sud-américaine. La mise en production d'un tel territoire au détriment de quelques milliers d'Indiens nomades ou seminomades, est en grande partie l'œuvre des capitaux et d'immigrants d'origine britannique, auxquels il faut néanmoins ajouter les autres migrants, des Allemands, Wallons, Basques, Boers, Levantins, Français et Espagnols. La diversité des origines des colons a entraîné la formation d'une société cosmopolite et constitue un clair exemple de front pionnier délogeant la société indienne originaire et ce n'est qu'assez récemment que la justesse de ce violent remplacement culturel a été remise en cause. Peu à peu s'est établie une «sheepocracy " pour reprendre le terme malouin qui exprime bien une société stable axée sur la filière ovine.

La société patagonienne s'est donc fondée sur l'élevage de moutons; de même que la territorialisation actuelle de l'espace qui découle directement de cette époque. Pourtant, on constate que «l'establishment anglo-pastoral » construit autour du mouton commença assez vite à subir l'érosion par le bas, avec notamment la révolte sociale des immigrés les moins bien lotis, et par le haut au travers de la pression nationaliste des gouvernements argentin et chilien.

Le temps passant, le mouton a commencé à perdre du terrain face à d'autres secteurs d'activité à qui les politiques publiques ont successivement confié les rênes du développement régional, tout d'abord le secteur énergétique avec le pétrole, le charbon et l'hydroélectricité, puis le tourisme et plus récemment l'exploitation minière. Même si en ce début de $\mathrm{xxI}^{\mathrm{e}}$ siècle, d'une part, la participation de l'élevage ovin au PIB régional n'atteint pas $5 \%$ et, d'autre part, environ $95 \%$ de la population est urbaine, le mouton demeure un élément identitaire majeur à l'échelle régionale et constitue le principal support de la gigantesque emprise foncière constituée par les parcours, et cela des deux côtés de la frontière Chili-Argentine. De plus, si l'élevage ovin est aujourd'hui fortement dévalué, notamment en lien avec la dégradation irréversible d’une grande part des parcours du côté argentin, le mouton reste une icône de la Patagonie, autant par son rôle structurant un siècle auparavant, que comme la seule activité qui assure l'occupation de l'ensemble du territoire.

\section{Que le mouton soit, et le mouton fût!}

À partir de sa découverte au XvI ${ }^{e}$ siècle, la Patagonie a occupé une position périphérique dans l'empire espagnol et les tentatives de s'y établir durablement ont échoué pendant toute la période coloniale. À la même époque, à cause de sa position sur le passage 
interocéanique, la Patagonie était fréquentée, et convoitée, par les Britanniques. Le vide géopolitique s'est prolongé jusqu'au début du xix ${ }^{e}$ siècle, au moment de l'indépendance des deux républiques sud-américaines qui pouvaient avoir des prétentions territoriales: l'Argentine et le Chili. C'est à cette période que les Britanniques réussirent à s'installer dans la région en délogeant, en 1833, un établissement argentin aux îles Malouines. En fait, à partir de la fondation de Port Louis en 1764 par Antoine de Bougainville, l'archipel avait connu une occupation presque ininterrompue, française d'abord, anglaise -clandestine- après, espagnole ensuite, et argentine enfin. Cette permanence dans l'occupation des îles Malouines fait contraste avec la situation sur le continent adjacent, qui demeura inoccupé par la « civilisation » et aux mains de quelques milliers d'Indiens nomades, les Tehuelches (ou Patagons). Seul à l'extrême nord de la région, sur le fleuve Negro, tenait bon le village de Carmen de Patagones, établi par les Espagnols en 1779. Ainsi, Carmen au nord et les Malouines au sud étaient les deux seuls points, côté Atlantique, d'où pouvait partir la colonisation de la Patagonie.

\section{San Gregorio}

L'état d'abandon des anciens bâtiments de l'Estancia San Gregorio, sur le Détroit de Magellan, reflète la profondeur de la chute de l'empire ovin, même si dans ce cas les causes de l'abandon n'ont pas été économiques ni agronomiques. Nombre d'estancias, grosses exploitations d'élevage extensif, ont été fermées partout en Patagonie, mais la décrépitude et le silence de ces installations abandonnées qui ont été jadis à l'origine d'une des plus grosses fortunes régionales

-celle de l'asturien José Menéndezsont spécialement frappants.

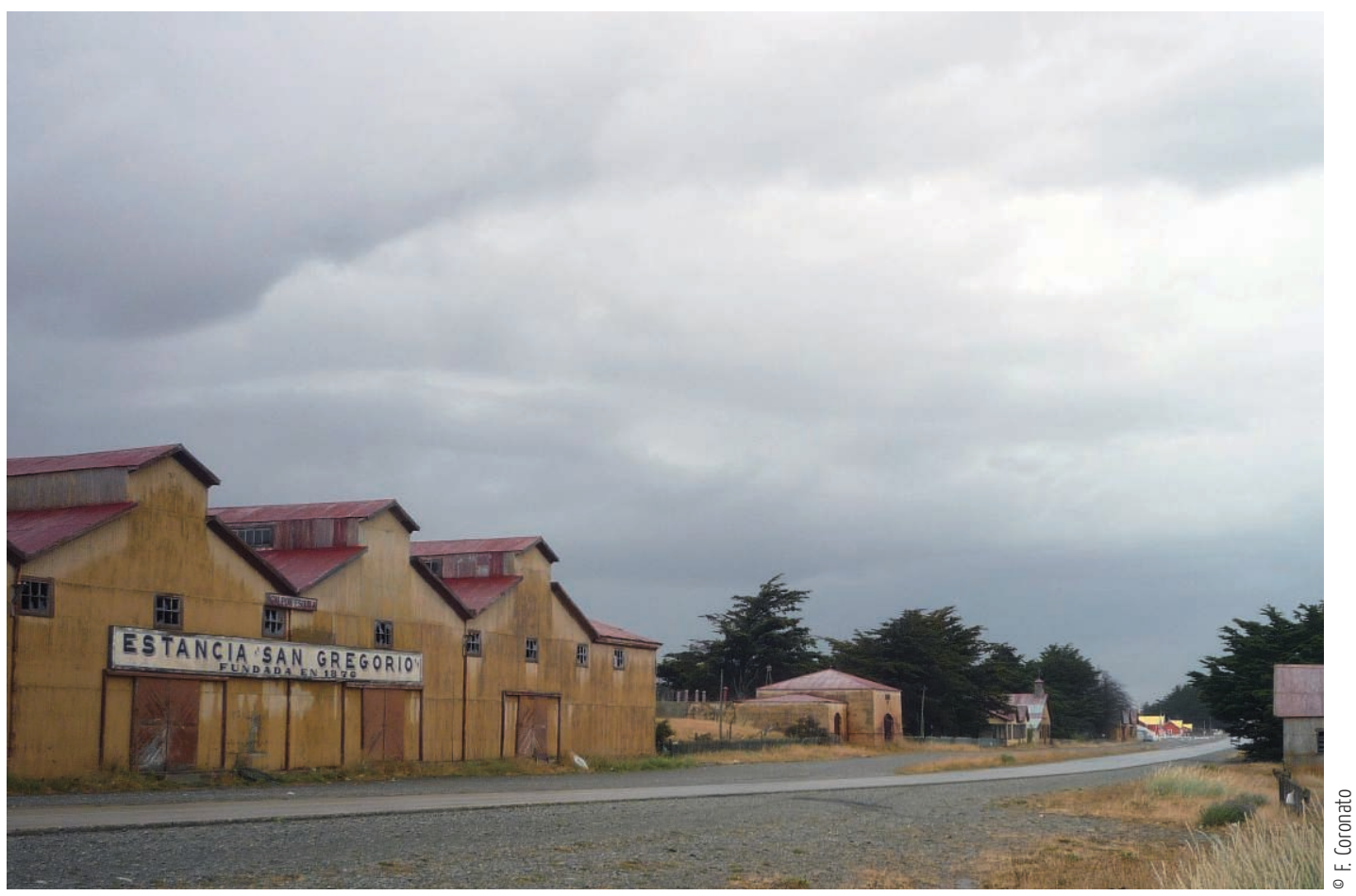

Sur la façade pacifique, les premiers colons à s'établir durablement dans la région sont les Chiliens qui, en 1843, forment un pénitencier à Punta Arenas, sur le détroit de Magellan. L'établissement allait végéter pendant quelques décennies jusqu'à ce que la navigation à vapeur convertisse le détroit de Magellan en route maritime obligée. Punta Arenas est alors devenue une escale incontournable de la navigation interocéanique. 


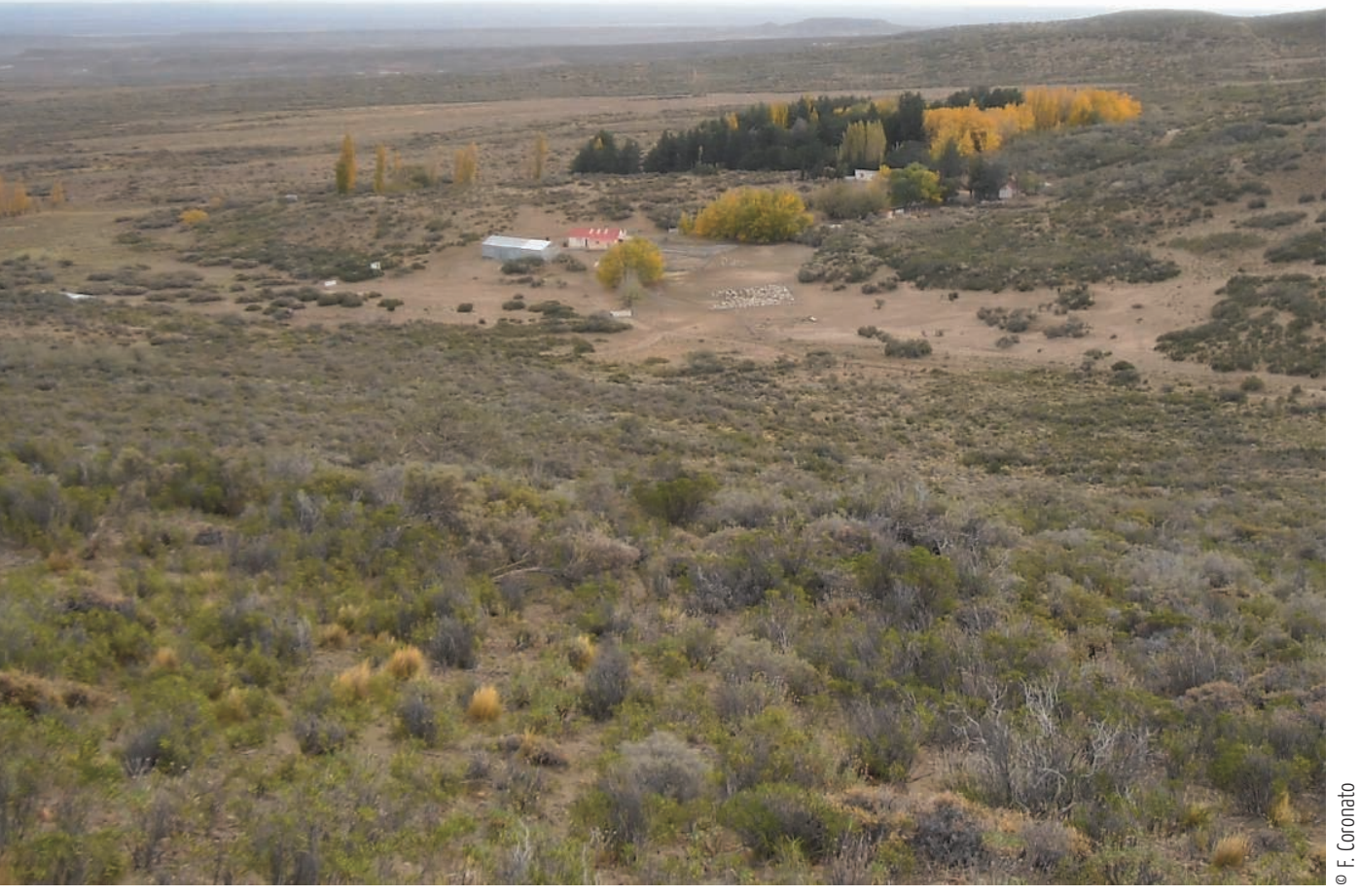

Au lointain

Les steppes de Patagonie sont naturellement dépourvues d'arbres. La présence de ceux-ci est toujours liée à la colonisation et permet de repérer les habitations au lointain. Arbustes et herbes prédominent à tour de rôle selon les conditions écologiques, ou cohabitent en formant une mosaïque favorable aux moutons.

Tous ces composants du paysage patagon apparaissent sur cette photo, y compris la vastitude. de laine passait de 10000 à 63000 tonnes. dans l'histoire écono-

mique argentine, la période 1850-1870 est connue comme celle de « la fièvre de l'ovin » ou encore de «la mérinisation », en lien avec la race Mérinos utilisée pour sa laine. Les sept millions d'ovins de 1850 sont passés à 41 millions en 1870, tandis que la production

L'essor du mouton est arrivé jusqu'aux Malouines où les conditions écologiques étaient non seulement favorables, mais surtout où il n'y avait pas de prédateurs, soient-ils animaux ou humains. La conjonction des deux fit qu'en trente ans, l'archipel atteignit sa saturation ovine: 800000 têtes. Pendant que les moutons foisonnaient dans la Pampa et les Malouines, la Patagonie en demeurait dépourvue, car les Chiliens de Punta Arenas et les Gallois qui s'étaient établis au Chubut en 1865 n'avaient que des troupeaux de subsistance. Hormis ces deux derniers points et Carmen de Patagones, la région entière appartenait aux Amérindiens.

La colonisation de la région démarra après la campagne de l'armée argentine contre les indigènes, connue comme "Conquête du Désert » (1879-1884), et appuyée par le consulat britannique qui voyait dans la Patagonie une extension possible de l'élevage ovin malouin et pampéen. Une fois la population native soumise et la sécurité assurée, l'ensemble de la Pampa et la Patagonie se sont ouverts à la colonisation agricole par le mouton. Mais l'emprise spatiale sur la région de la part du gouvernement était bien loin d'être complète alors; en fait, les colons eux-mêmes ont maintes fois été les tout premiers à prendre contact avec un territoire et ses habitants autochtones, et pas toujours pacifiquement; il y en a qu'y voit une conquête sous-traitée. Notons dès à présent que si les conditions climatiques de la Pampa, humides et tempérées, ont permis d'y établir très tôt un élevage bovin de qualité et de grandes cultures céréalières, en revanche la Patagonie, à cause de son environnement austère, aride et froid, avec des champs de parcours moins productifs, n'eut que l'ovin pour s'insérer dans le marché mondial. 


\section{L'outil de la territorialisation}

Dans la seconde moitié du XIX ${ }^{e}$ siècle, à l'époque où l'Europe demandait davantage de matières premières pour soutenir l'essor de son industrie, qui -à son tour- produisait un excédent démographique, les pays tempérés de l'hémisphère sud se sont insérés dans ce système globalisé en tant que fournisseurs d'intrants et récepteurs de migrants et capitaux. La colonisation ovine de la Patagonie se place donc dans ce contexte international d'expansion des frontières agricoles, ce que dans le contexte national argentin s'est manifesté par l'élargissement des frontières politiques.

On peut considérer que l'avancée sur de nouvelles terres en est à la fois une cause et une conséquence. En effet, s'il est vrai que le boom ovin dans la Pampa dans la deuxième moitié du XIX ${ }^{e}$ siècle a fait pression sur le déplacement de « la frontière » vers la Patagonie, il est aussi vrai que la Conquête du désert, mentionnée précédemment, a mis à la disposition du gouvernement argentin et de ses alliés britanniques d'immenses étendues, qu'on s'empressa de remplir de moutons, d'autant plus qu'ils étaient déjà en surnombre aux Malouines et limitaient les grandes cultures dans la Pampa.

Ainsi, le mot d'ordre du gouvernement argentin a été: « la colonisation de la Patagonie doit partir de Carmen de Patagones et avancer aux pas des troupeaux », une expression du président Avellaneda en 1880 qui fait l'exacte pendant de celle du président brésilien GarrastazuMédici presque un siècle plus tard à propos de la colonisation de l'Amazonie par la patte du bœuf. La même stratégie d'occupation de l'espace patagonien pour le mettre en valeur a été mise en œuvre par les Britanniques, d'une part à partir des Malouines et d'autre part à partir de Punta Arenas avec leurs alliés chiliens.

C'est ainsi qu'à partir des années 1880 les moutons sont entrés en masse en Patagonie en provenance du nord et du sud, c'est-à-dire de la Pampa et des Malouines, directement ou via Punta Arenas. S'ils quittaient la Pampa sous la poussée des bovins et des cultures céréalières, ils quittaient les Malouines en raison de la surpopulation ovine. Rien de plus évident pour ces éleveurs îliens, que l'avantage de s'installer dans des parcours vierges situés à deux ou trois jours de bateau. Les premiers troupeaux, à titre de test, ont été parqués sur une petite île du Détroit de Magellan en 1876, et vu la réussite, l'expérience s'est généralisée dès le début de la décennie suivante. Au même temps, au Nord, à l'autre extrême de la région, une fois que la campagne militaire désenclava Carmen de Patagones et permit la communication terrestre entre

\section{Lafonia}

Le boom ovin dans les Malouines a devancé de 20 ans celui de Patagonie, en partie grâce à l'absence de prédateurs et aux avantages climatiques. Cependant, la filière ovine s'est développée davantage sur le continent, notamment par des questions d'échelle et de marché, tant régional comme international. Quant à la gestion des moutons eux-mêmes, il y a très peu de différences entre les îles et le continent, et les méthodes de tonte et reproduction sont quasiment les mêmes, aussi que les mœurs des hommes qui s'en occupent et le poids identitaire dans la population locale. Sur la photo, des moutons malouins viennent d'être tondus, début décembre, en Lafonia (sud de l'île de l'Est). 


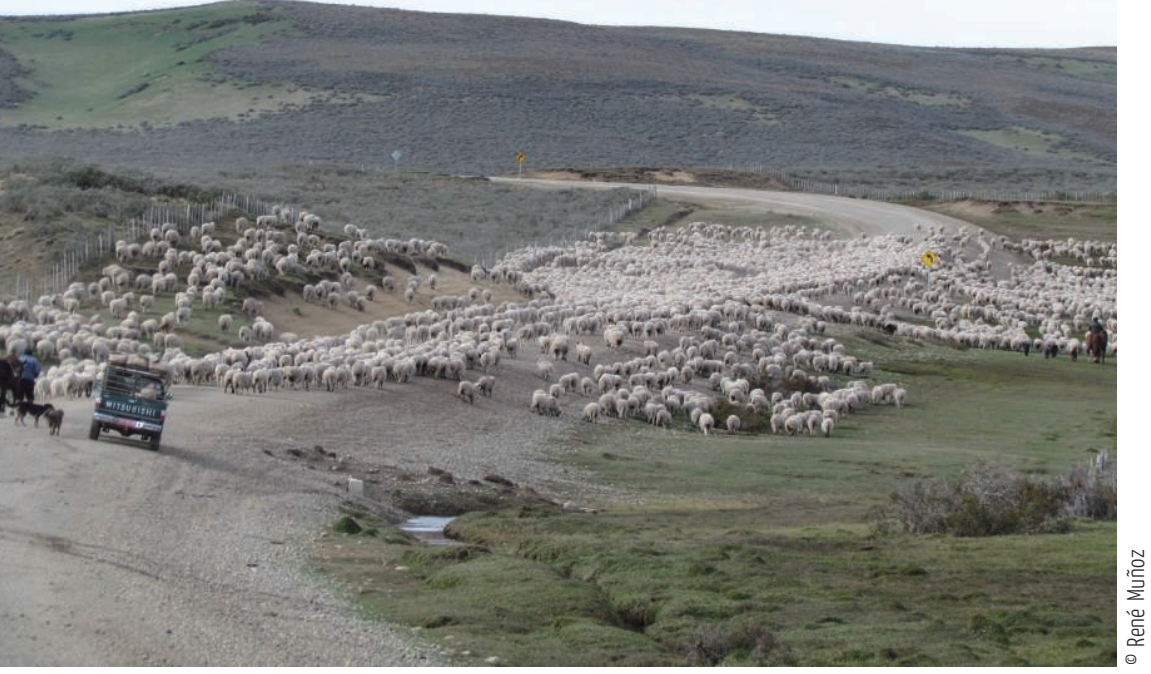

Arreo

Si dans la plupart de la Patagonie les troupeaux demeurent toute l'année sur les mêmes parcours, dans les champs les plus hauts (700-1100 m) et à proximité de la cordillère des Andes l'alternance estivage/hivernage s'impose.

Pourtant l'hivernage ne comporte pas de bâtiment mais juste des parcours plus bas et abrités. Les déplacements de bétail (en espagnol: arreo) entre les champs d'hiver et les estives peuvent devenir encombrants s'ils empruntent les chemins publics.

Au début de la colonisation de la région,

les arreos en départ de la Pampa pouvaient comporter des milliers de moutons et prendre plus d'un an.
Buenos Aires et le Rio Negro, les ovins se sont rapidement répandus vers le sud. L'expansion a été en tache d'huile sur les terres avoisinantes, ou "transplantée » vers des points situés bien plus au sud, moyennant de longs déplacements des milliers de moutons à travers la steppe (les arréos); certains sont devenus légendaires, comme celui de 5000 moutons conduits par MM. Jamieson, Saunders, Hamilton et Mac Lean en 1887, qui a mis plus de deux années avant d'arriver (Dobrée, 2006).

Il y a ainsi eu deux fronts de peuplement. En repoussant aux confins de son territoire la société préexistante et en restructurant totalement son espace, ces fronts peuvent être considérés comme des fronts pionniers selon la définition donnée par Poccard (2004). Les deux étaient dynamisés par le besoin de parcours pour l'élevage ovin. Cependant si le front du nord avait une forte composante nationale argentine, hispanisante et catholique, celui du sud était plutôt cosmopolite, laïcisant et anglophile. Les deux groupes ont pourtant commis la même erreur de surestimer la productivité des parcours patagoniens. En effet, les Falklanders (Malvineros ou Malouins) débarquant leurs troupeaux sur les terres arrosées du détroit de Magellan retrouvaient un paysage fort ressemblant à celui des Malouines, à la productivité comparable sinon meilleure. Les moutons se sont acclimatés sans difficulté à ce nouvel environnement dont le seul vrai changement était le danger causé par la présence des prédateurs, que ce soient des pumas ou des Amérindiens. En revanche, dès qu'ils sont remontés une centaine de kilomètres au nord, la pluviométrie nettement plus faible ne donnait pas les mêmes parcours. Ils n'en ont pas tenu compte. Ils ont adopté des charges comparables proches d'un ovin par hectare, voire plus, sans se rendre réellement compte qu'ils surexploitaient et donc dégradaient lentement mais sûrement leur ressource pastorale, en particulier la composante arbustive de la steppe patagonne, garante de la survie de la composante herbacée et de l'activité biologique du sol. Puis, à la faveur d'une, deux ou trois années de moindre pluviométrie, une érosion éolienne un peu plus poussée associée à quelques pluies battantes, la steppe est devenue un glacis par pans entiers à l'échelle des exploitations et de la région.

Dans le même temps, les éleveurs qui pénétraient en Patagonie par le nord en conduisant des troupeaux pampéens, avançaient sur un pays des plus monotones, où le relief absolument plat accentue la gradualité d'un écotone qui s'étale sur des centaines de kilomètres. Ainsi ces moutonniers - pour la plupart d'origine basque - ne percevaient pas bien que les changements dans la ressource pastorale demandaient des changements équivalents dans la gestion des troupeaux, notamment des charges moins fortes et des temps de récupération plus longs, en particulier pour la composante arbustive.

Par ailleurs, conforme aux descriptions faites par les premiers explorateurs de l'intérieur, on peut dire que l'essor initial des moutons en Patagonie reposait aussi sur l'abondance de biomasse qu'offraient les parcours jusqu'alors vierges de tout pâturage systématique. Ceci étant vrai pour l'ensemble de la région et a constitué, donc, un capital qui a 


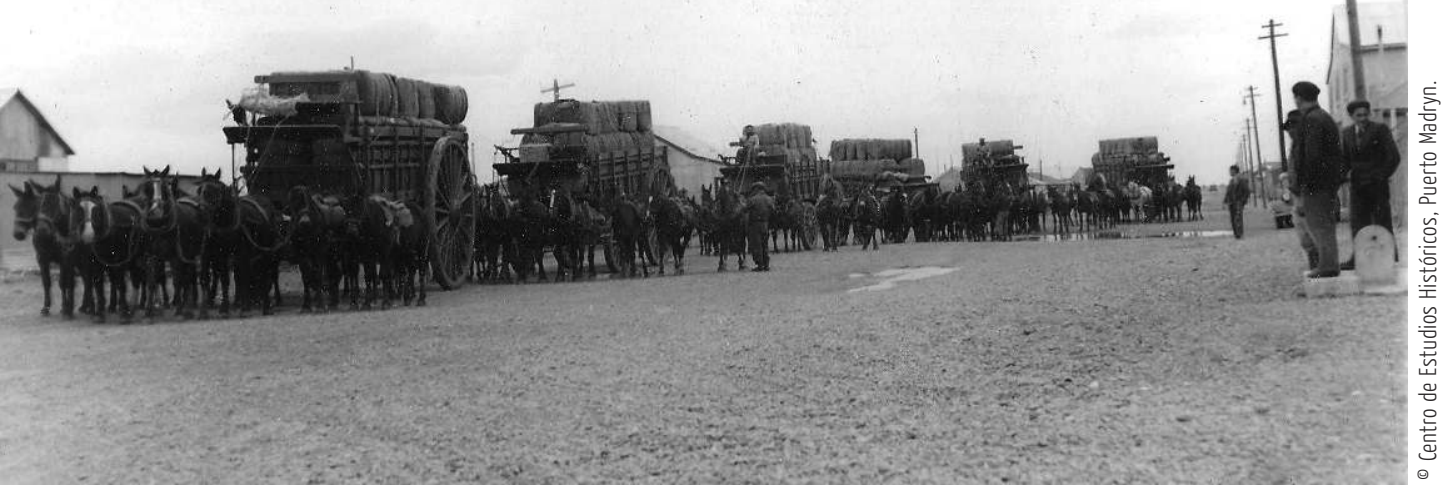

profité aux deux fronts pionniers. Le fait que ces parcours steppiques, plus résistants que résilients, cèdent de manière soudaine sous une trop forte pression pastorale conjuguée à quelques effets climatiques, est à mettre également à la décharge des pionniers qui ignoraient leur fonctionnement.

Par conséquent, au début, les éleveurs du nord et du sud se sont trompés sur les capacités productives de leurs parcours et les ont surexploités. Les charges ovines du début de la colonisation (environ un mouton par hectare en moyenne) n'ont forcément pas pu durer une fois que la biomasse initiale fut épuisée et que l'on avança sur les parcours plus arides de l'intérieur. Le mirage fut prolongé par la Première Guerre Mondiale (19141918) qui fit monter en flèche le prix de la laine et de la viande ovine. C'est au cours de cette période faste que la plupart de l'intérieur aride fut occupé; la mise en exploitation hâtive de ces terres marginales tourna court avec, à partir des années 1960, une sévère dégradation allant jusqu'à la désertification.

\section{La politique du " boom and crash » et ses conséquences écologiques}

Au commencement de la colonisation, les erreurs dans la perception des capacités des parcours de Patagonie n'étaient pas seulement issues de l'ignorance du fonctionnement des écosystèmes mais aussi de la spéculation foncière pure et simple. D'ailleurs, la surestimation des parcours et l'euphorie qui en découla chez les

\section{Transport}

L'acheminement de la laine à dos de cheval au début, puis en charriots, et en camions à partir des années 1930, empruntait les sentiers indiens qui allaient devenir des pistes. Le réseau routier de Patagonie a été, en grande mesure, façonné pour le transport de la laine aux ports, de même que les quelques chemins de fer, aujourd'hui disparus. Les caravanes de charriots tractés par des mules avançaient $30 \mathrm{~km}$ par jour, et mettaient donc 20 ou 30 jours pour faire la traversée depuis les Andes à l'Atlantique. Les balles de la photo haut indiquent que les estancias desservies avaient une presse hydraulique, tandis que les producteurs de la photo bas n'en avaient pas et devaient se contenter de livrer leur laine en ballots.
- Centro de Estudios Históricos, Puerto Madryn.

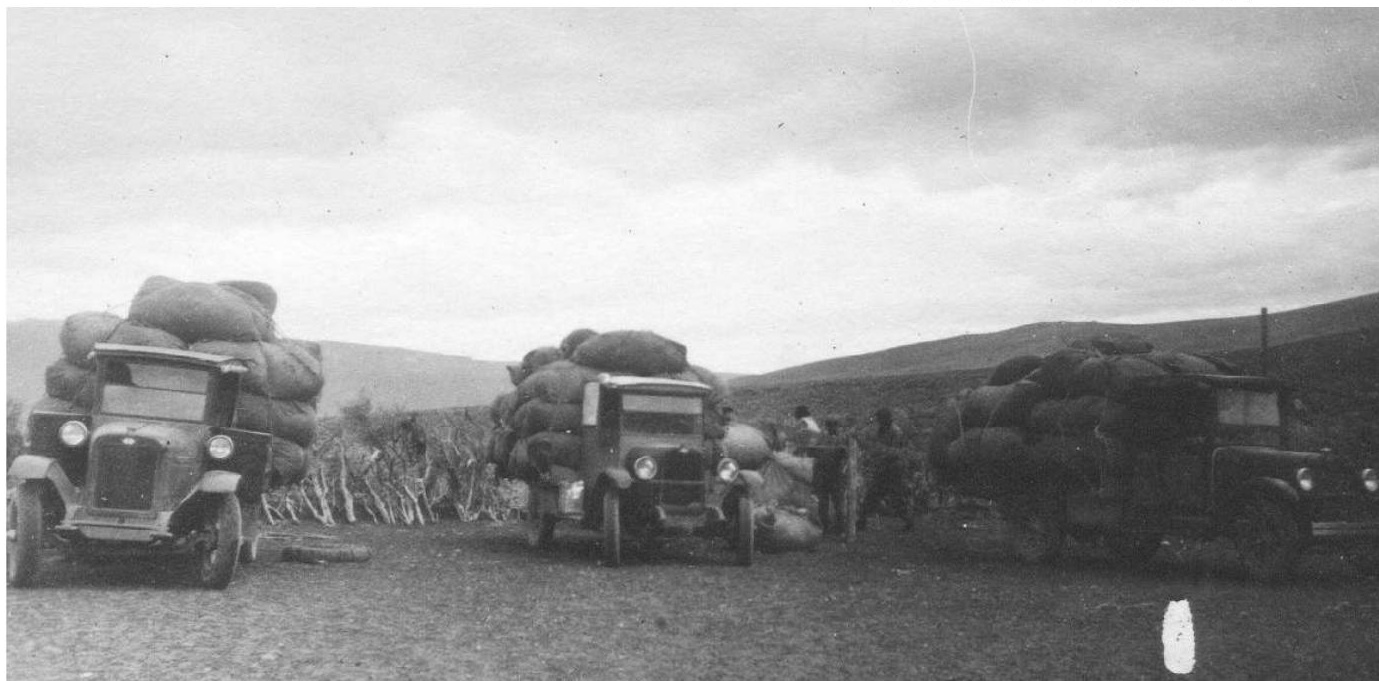




\section{La colonisation par les ovins}

Les trois foyers de dispersion des ovins en Patagonie à la fin du xixe siècle, Carmen de Patagones, Punta Arenas et les Malouines, répondaient en théorie à trois gouvernements différents (respectivement, Argentine, Chili et Grande-Bretagne). Loin de se concurrencer, la synergie a conduit à une même région transfrontalière organisée le long de la filière ovine, et dont la capitale économique et sociale était Punta Arenas, attirant des capitaux de Santiago et Buenos Aires mais surtout des capitaux liés à l'industrie textile européenne. Les troupeaux provenant du nord étaient surtout de race Mérinos, tandis que ceux du sud comportaient des animaux de race Lincoln d'abattoirs frigorifiques exportaient vers l'Europe au début du $x x^{e}$ siècle. Le déclin de l'industrie frigorifique à partir des années 1950 accéléra la crise environnementale car les exploitations, misant dorénavant sur la laine, ont commencé à surcharger leurs parcours. C'est à cette époque que la Patagonie atteignit vingt millions d'ovins. L'occupation du territoire fut alors complète même si la population de l'ensemble de la région ne dépassait pas les 400000 habitants. Par la suite, l'élevage ovin commença son déclin. Aucune activité économique alternative nassura la mise en valeur de l'ensemble de la région. Pétrole, industrie, agriculture, tourisme et pêche ont connu et connaissent encore un essor significatif, mais leur localisation particulière et leur emprise limitée laissent d'immenses espaces vides de peuplement où seul le mouton résiste. et puis Corriedale. Le sud s'est tourné plus vers la viande. Une douzaine

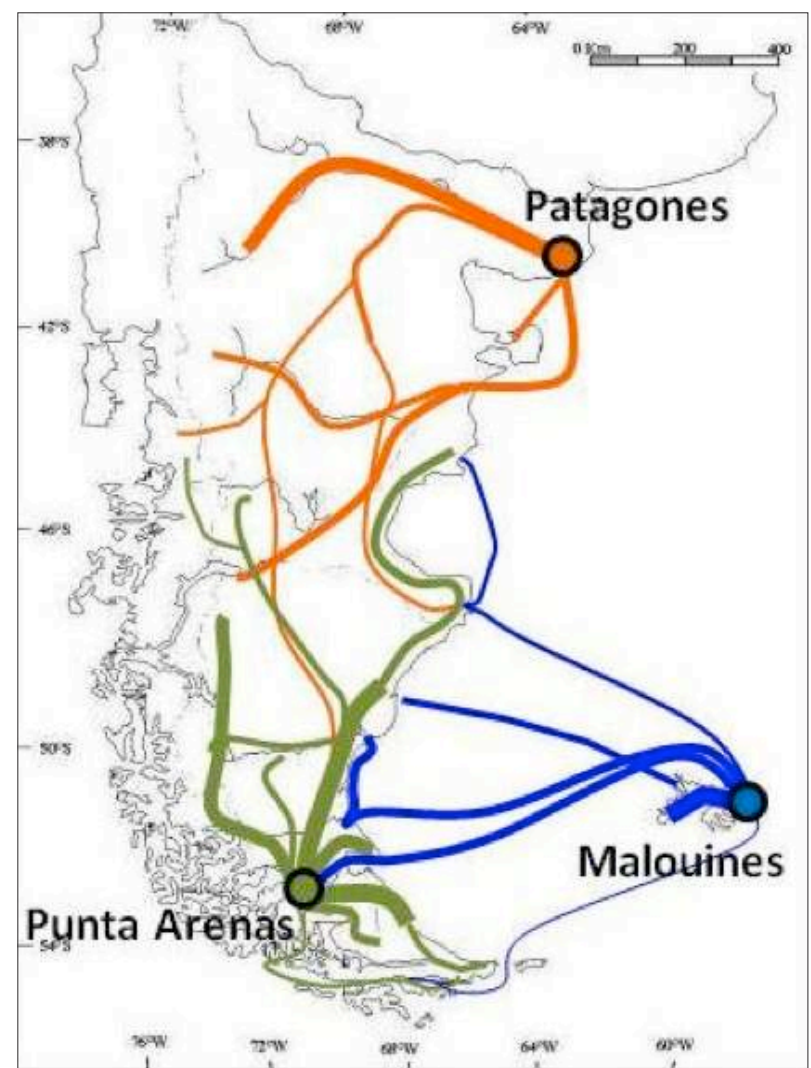

Les données agronomiques

Les isohyètes permettent d'estimer rapidement le Coefficient d'Efficacité Pluviale (CEP), c'est-à-dire la production primaire (en kg de matière sèche, MS) produite par hectare par millimètre de pluviométrie annuelle. Dans les steppes de Patagonie on estime un CEP maximum de l'ordre de 4 pour un parcours bien conservé - situation que nous supposons était la condition des pâturages au début de la colonisation ovine. En ce temps-là, la Patagonie aurait présenté une production primaire d'environ $800 \mathrm{~kg} \mathrm{MS/}$ ha/an. Supposant un taux d'utilisation de la végétation de $50 \%$ cela fait $400 \mathrm{~kg} \mathrm{MS} / \mathrm{ha} / \mathrm{an}$, et si l'on estime l'ingestion annuelle d'un mouton en $335 \mathrm{~kg}$ MS, il s'ensuit que la capacité de charge des parcours patagons « au départ » aurait été grosso modo de 1,2 mouton par hectare. Si l'on refait ces calculs avec une pluviométrie de $300 \mathrm{~mm}$ (celle des régions où l'élevage a démarré), on arrive à 1,8 mouton par hectare. Ces résultats sont peut-être surestimés, mais ils permettent d'expliquer l'euphorie initiale et la chute qui en suivit. II suffit que le CEP diminue à 3 et ramener le taux d'utilisation aux maxima actuels (30\%) pour que la capacité de charge tombe à 0,8 ov/ha, et cela dans les zones les mieux arrosées de la Patagonie.

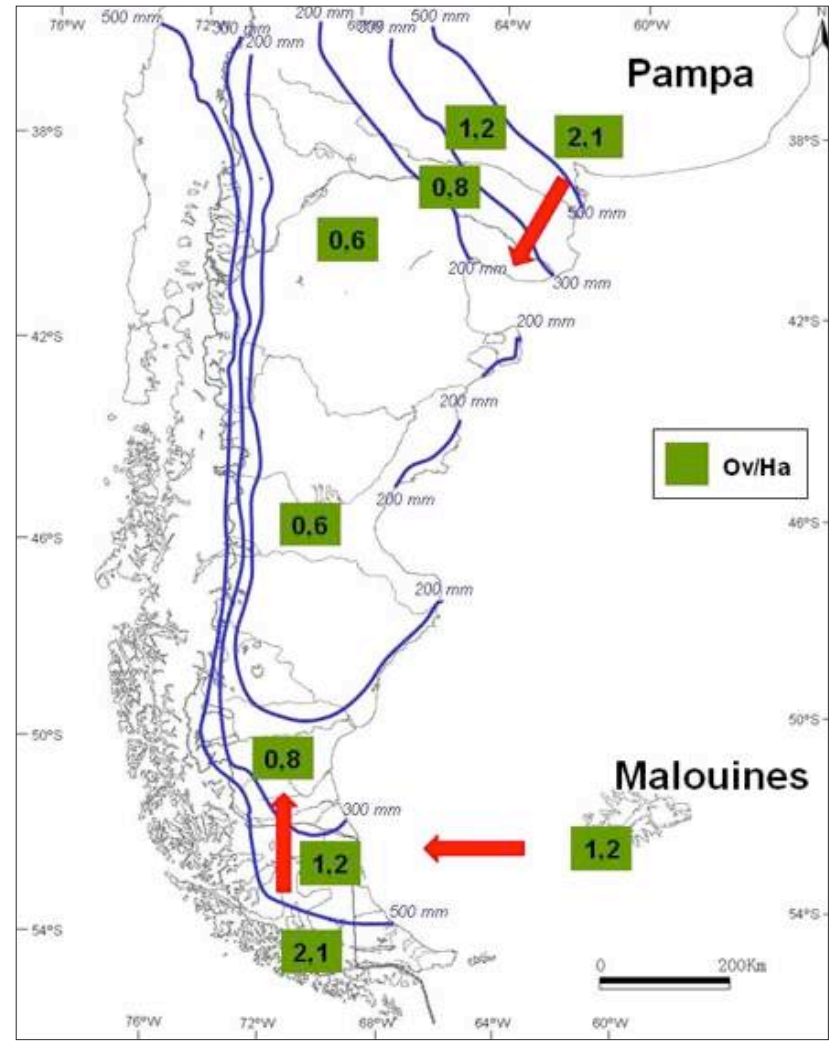


moutonniers, n'ont pas été les seules responsables de la détérioration des pâturages, qui commença à se manifester vers les années 1950 sans être prise au sérieux. Ici, on est aux antipodes du système lapon, soucieux de préserver la ressource et tenu de s'y adapter de façon très fine (voir l'article de Samuel Roturier dans ce volume). Loin de là, les politiques publiques mises en route à la hâte vers 1890 afin d'occuper sans plus attendre la nouvelle conquête du « colonialisme républicain » (Obregón \& al., 2011) ont leur part de responsabilité. Tout d'abord, le cadastre orthogonal sur lequel est toujours établi le foncier patagonien, a été directement transposé des plaines de la Pampa. Or en Patagonie les contraintes en eaux et climatiques sont autrement plus importantes que dans la Pampa plane et bien arrosée. Ensuite, dans sa volonté d'occuper au plus vite la région, le gouvernement a favorisé la mise en place d'un système latifundiaire. Les premiers colons se sont ainsi constitués d'immenses domaines fonciers, de surcroît sur les meilleures terres, et cela avant 1900, laissant aux suivants les terres les moins productives et de moindre surface en lien avec la législation plus restrictive en termes d'attributions.

Ensuite, à partir des années 1920, l'impossibilité légale d'accéder au foncier en tant que propriétaire n'a pas favorisé la gestion durable des parcours. C'est même le contraire qui s'est produit dans la mesure où a prévalu la stratégie du profit immédiat au détriment de la durabilité de la ressource,

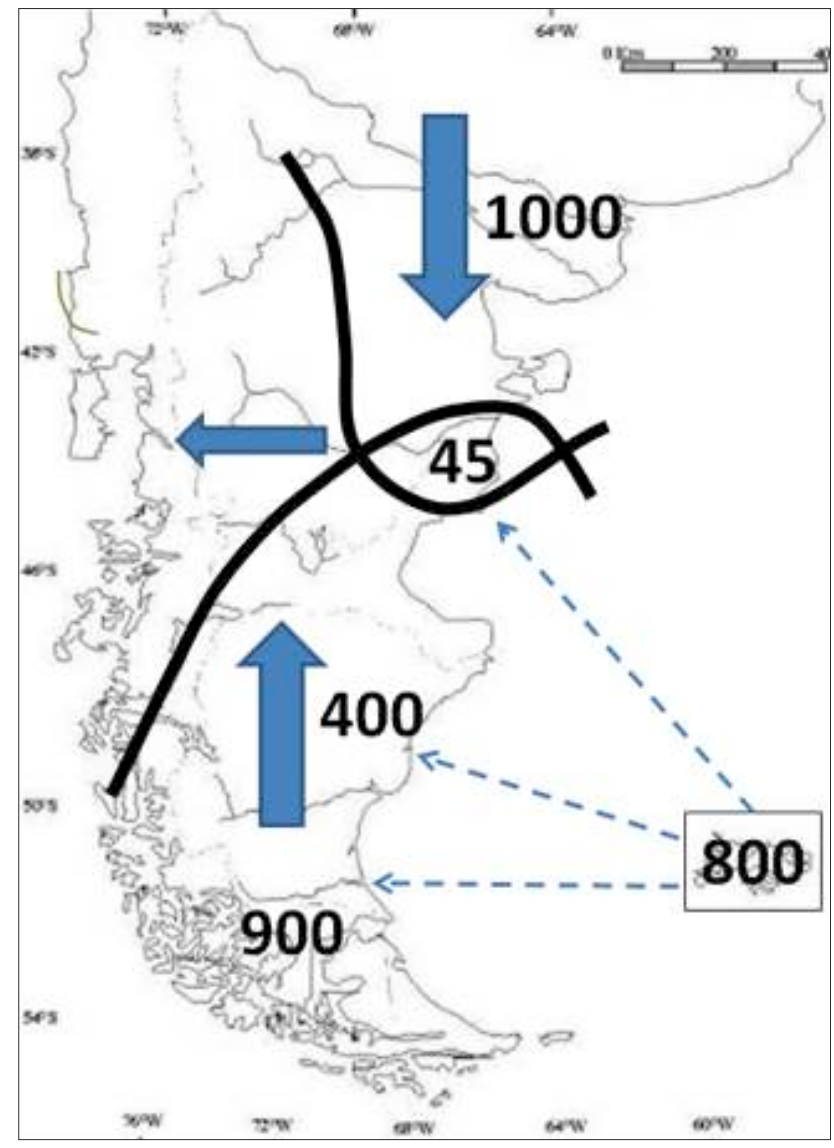
d'autant plus que les colons étaient seulement locataires des terres publiques. Pour l'immense majorité des exploitations qui avaient démarré à la fin du boom lainier, l'acte de propriété n'est venu qu'en 1958, lors de la provincialisation des territoires nationaux. Mais il était déjà trop tard! L'érosion et la désertification étaient déjà bien avancées, trop avancées.

De cette manière, la gestion inadéquate des troupeaux ovins serait responsable de la dégradation sévère des parcours patagoniens. Il y a de vastes zones de Patagonie où la ressource pastorale semble irrécupérable. Del Valle et al. (1997) ont évalué que le quart des zones arides de Patagonie présentent un niveau « sévère » de désertification et que celle-ci atteint un niveau « très sévère » dans près d'un dixième de la région. C'est-à-dire qu'un tiers des steppes de Patagonie est désormais incapable d'être exploité en élevage ovin, tel qu'on le conçoit à présent. Ceci explique l'abandon de nombreuses exploitations, triste phénomène affectant les zones les plus défavorisées du point de vue climatique. Dans la province de Santa Cruz, la plus touchée, plus de 300 exploitations (sur un total de 1260) étaient fermées en 1991. Leur nombre dépassait les 500 en 1997 (Andrade, 2005 : 689) et les 600 en $2014^{2}$. À l'heure actuelle, l'élevage durable sur des parcours naturels est possible seulement dans certaines parties de la Patagonie, en particulier l'extrême sud et le long de la Cordillère andine. Une bonne part du centre de la région est a priori condamnée car il n'existe pas de solutions viables pour reconstruire, puis maintenir une production durable (Noy-Meir, 2005 : 204). Le climat n'est pas le seul facteur naturel impactant négativement l'élevage ovin en Patagonie. En effet, en 1991, 2008 et 2011 il y a eu des éruptions volcaniques dans les Andes du Sud, qui ont rejeté une quantité très

\section{Les fronts pionniers}

Les troupeaux partant de la Pampa, passaient par le Chubut pour rejoindre Santa Cruz. Mais si - d'après le recensement de 1895 - il y avait 45000 moutons au Chubut, il y en avait 400000 à Santa Cruz. Cette différence montrerait combien a été importante pour le territoire de Santa Cruz l'introduction ovine provenant des Malouines et de Magellan.

En effet, en 1895 il y avait 900000 ovins à Magellan et en 1898 les Malouines ont atteint leur maximum historique, soit 807000 d'après Bernhardson (1989). La supériorité numérique du cheptel de Santa Cruz par rapport à celui du Chubut (même si les deux territoires ont été ouverts à la colonisation au même moment) s'expliquerait non seulement par la proximité du centre de dispersion des Malouines et l'environnement plus favorable aux moutons, mais, surtout, par la main-d'œuvre spécialisée que représentaient les moutonniers Malouins, spécialisation qui manquait aux Gallois du Chubut et qui fut remplacée en partie par les Basques venant de la Pampa. 


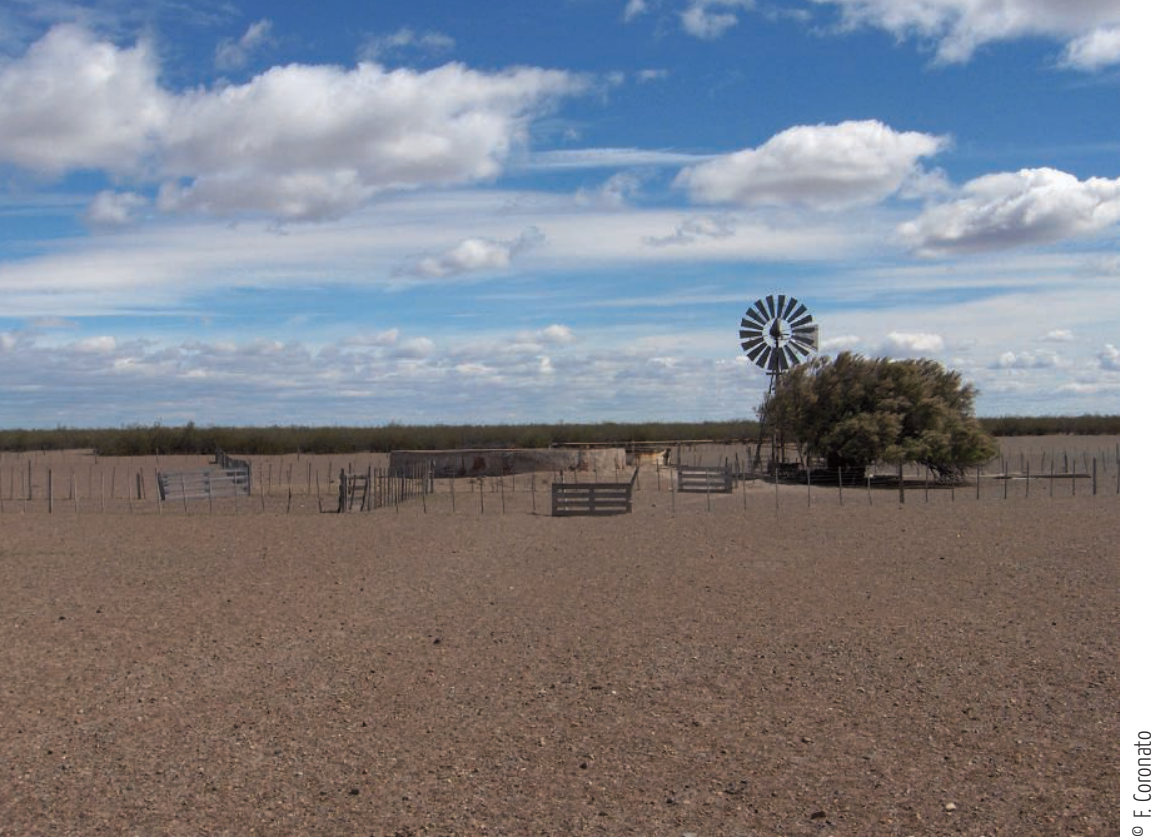

\section{Les Puits}

Fort souvent, dans le chaparral du nord-est de la Patagonie, les exploitations de dix mille hectares n'ont qu'un seul point d'eau au centre. Ceci entraine de longs déplacements journaliers pour les moutons, mais aussi des différences marquées dans l'utilisation du pâturage en allant du centre à la périphérie des parcours. Le surpâturage près du point d'eau va de pair avec le sous-pâturage aux bords des champs. Dans cette région, les points d'eau sont des puits pouvant dépasser les cent mètres de profondeur, creusés à la pioche et à la pelle par les pionniers d'il y a un siècle, des basques pour la plupart. importante de cendres toxiques. Les vents d'ouest se sont chargés de les répandre sur toute la Patagonie, jusqu'à la côte atlantique, en entraînant la mort de plusieurs centaines de milliers de moutons. Les survivants ont été affectés dans leur santé et productivité et dans la qualité de leur laine, de même que la qualité de la vie des moutonniers. Ce fut le coup de grâce pour de nombreuses exploitations déjà au bord de la faillite.

Enfin, dans ce contexte socio-environnemental préoccupant, le déclin de l'industrie ovine en Patagonie a été aggravé à son tour par des facteurs extrarégionaux tels que l'instabilité des politiques économiques en Argentine, l'impact de la mondialisation, surtout à travers les grandes variations des prix internationaux des deux principales commodities qui sont la viande et la laine. Somme toute, il fallut que la filière ovine en Patagonie soit au plus bas vers l'année 2000 pour qu'un nouveau cycle s'amorce timidement. Il se présente comme un cycle plus sélectif, exigeant, écologique, où la valeur ajoutée et la qualité feront le tri entre gagnants et perdants; un cycle -enfin- où les concurrents du mouton, exploitation minière et écologie primaire, seront à l'affût.
Foin

Le contact entre les troupeaux (y compris bovidés) et les bois recouvrant les Andes pose des conflits à la conservation de la forêt. Encore qu'il s'agisse d'une zone plus productive que les steppes de plus à l'est, les hivers y sont plus rigoureux. Supplémenter avec du foin à l'entrée de l'hiver peut donner aux moutons l'énergie nécessaire pour survivre aux grands froids.

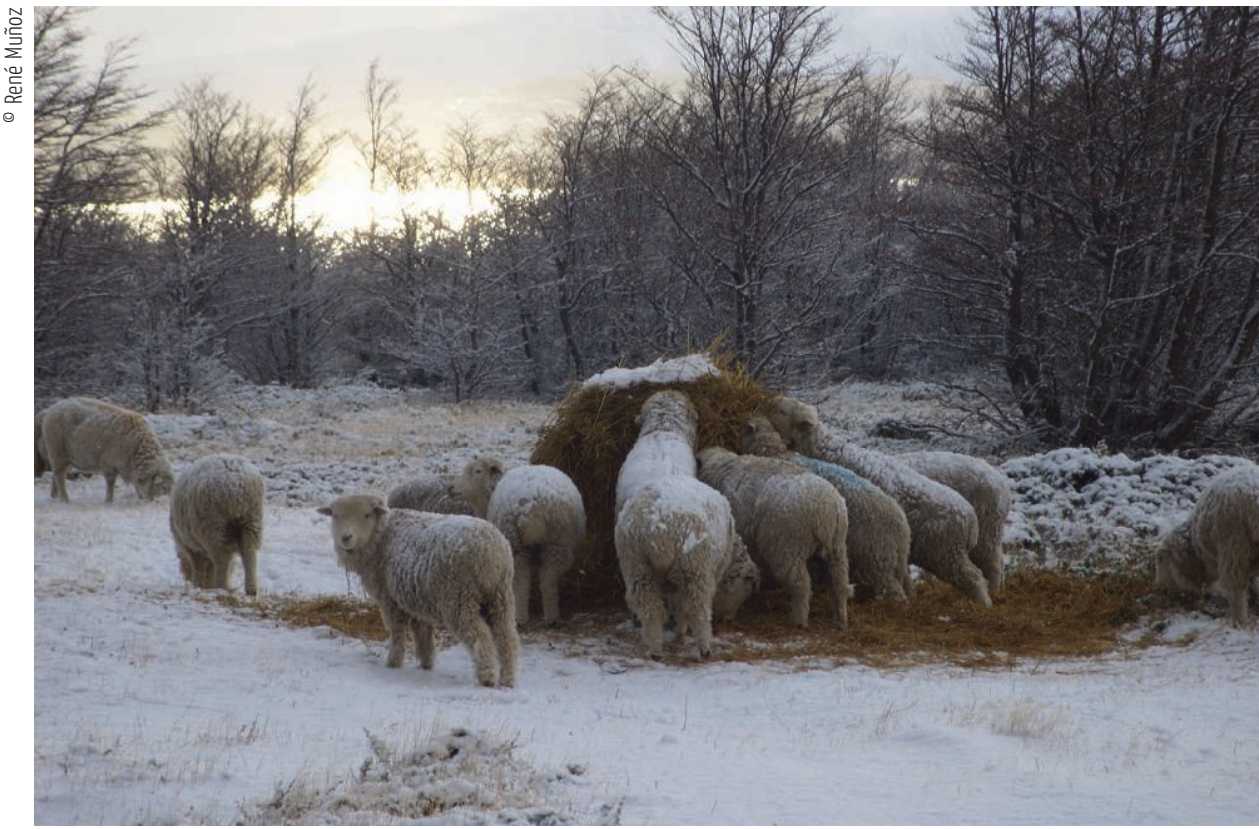




\section{Les arguments de l'insouciance}

Pourquoi a-t-on laissé sombrer ainsi l'élevage ovin? N'y a-t-il pas eu des réactions face au déclin?

Très tôt quelques voix se sont élevées, mais c'était l'époque du boom lainier de la Première Guerre Mondiale et de la reconstruction de l'après-guerre. Ainsi, dans l'euphorie générale, qui a prêté attention aux premières alertes sur le surpâturage, comme celle de Willis (1914) ou celle de Scott en 1919 (2001).

Or, le premier s'est surtout focalisé sur les conséquences économiques de la mauvaise gestion écologique, annonçant déjà les limites de la durabilité:

« Les parcours de Patagonie ont été exploités en vue du profit immédiat et ont produit de grandes richesses. À l'heure actuelle, plutôt qu'à la quantité de têtes - qui d'ailleurs est probablement proche de la capacité limite des pâturages-, on commence à faire plus attention à une gestion intelligente et prévoyante, qui vient remplacer le management improvisé d'antan. [...] L'éleveur doit rejeter la supposition irréfléchie que l'herbe repousse année après année même si elle a été broutée avant que les fleurs n'aboutissent en graines et dès que les jeunes pousses bourgeonnent après les premières pluies. Les fourragères, par conséquent, en perdent du terrain et les mauvaises herbes en gagnent. Ainsi s'opère le remplacement des herbes nutritives par des épineux amers. Tous les propriétaires devraient réaliser que leur véritable capital c'est la végétation, et que quand celle-ci s'appauvrit à cause de l'excès de moutons, c'est une perte qui doit être compensée. » (Willis, 1914, : 132; Traduction des auteurs)

Nous avons dit plus haut que les premiers signes évidents de la désertification sont apparus dans les années 1950, justement quand le nombre d'effectifs ovins atteignait son maximum. C'est à cette époque quand l'agronome Alberto Soriano commence à faire les premières observations scientifiques sur la santé des parcours. Tout en regrettant « l'absence de perception de la détérioration » chez les éleveurs patagoniens (Soriano, 2000 :30), on prêche restera longtemps confine dans le milieu académique. La pérennité des pâturages a commencé à attirer l'attention des éleveurs à partir des années 1980, et est devenue une question nationale lorsque la détérioration est devenue trop coûteuse en termes de diminution de la production. En ce qui concerne la laine, la diminution directement attribuable à la désertification aurait été de 0,5\% par an entre 1940-1987, et ceci dans des exploitations bien gérées et dans de bons parcours dans le nord-ouest du Chubut (Soriano \& Paruelo, 1990 : 51).

La réalité est l'absence de prise en compte de la ressource pastorale dans la gestion du troupeau, qui est concentrée sur la santé et la génétique des animaux, et cela depuis le début de la colonisation. La recherche agronomique sur les pâturages était presque

\section{Cendrillon}

Au cours des derniers 30 ans, les cendres issues de trois éruptions volcaniques des Andes du Sud ont couvert d'immenses étendues des plateaux de l'Est, en aval sous le vent. À chaque occasion, la mortalité ovine atteignit plusieurs centaines de milliers d'effectifs. Ces catastrophes ont été le coup de grâce pour les exploitations alors au bord de la faillite. 


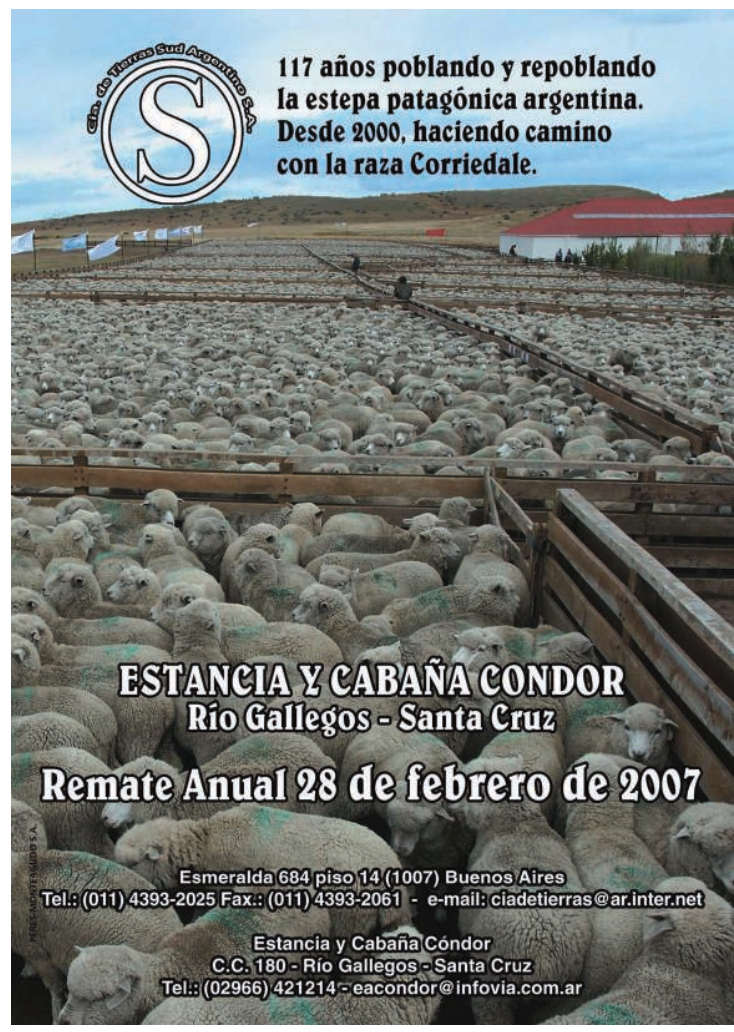

Enchères

Brebis et béliers voués à la reproduction sont vendus aux enchères dans des estancias spécialisées dans leur élevage (les cabañas). Ces véritables foires se tiennent à la fin de l'été (février-mars).

Ci-dessus, la publicité des enchères à l'Estancia Condor, la plus vaste de Patagonie, naguère propriété de la firme Benetton, un empire du textile en laine. Même si le Mérinos connaît un essor soutenu depuis plus d'un demisiècle, la race corriedale l'emporte dans le sud de la région, plus froide et moins aride. nulle en Patagonie et se concentrait dans la Pampa humide (Defossé \& Robberetch, 1987). De plus, il y aurait une fragilité intrinsèque des parcours de Patagonie, et d'une manière plus générale d'Amérique du Sud par rapport à ceux de l'hémisphère nord ou de l'Afrique australe. En effet, les pâturages du Cône Sud américain ont évolué sans la présence de grands herbivores, (les guanacos ne dépassent guère les $100 \mathrm{~kg}$ ). D’après Milchunas et al. (1988), du point de vue évolutif, ces pâturages n'ont pas eu l'occasion de développer des adaptations au prélèvement intensif d'herbivores, encore que Lauenroth (1998) estime possible une densité de 0,3 guanaco par hectare (équivalant à 0,6 mouton) avant l'introduction des ovins. Les steppes de Patagonie, plus résistantes que résilientes, se seraient très vite appauvries face au pâturage intensif des débuts de la colonisation (Soriano \& Paruelo, 1990).

\section{Remise en cause de l'ovin en Patagonie}

\author{
«Quand l'arbre est tombé, chacun court aux branches »
}

Considérant les points précédents, la remise en cause de l'ovin en Patagonie semble logique, notamment parmi les partisans de la conservation des écosystèmes. Cette idée est plus présente en dehors de Patagonie que dans la région elle-même, et se nourrit de l'aura mythique de grands espaces et nature préservée dont elle jouit à l'échelle internationale. Ainsi, comme si elle appartenait à l'humanité entière, tout comme l'Amazonie, et en faisant fi de la souveraineté des États et des décisions de ses habitants, diagnostics et propositions sur le destin de la région vont dans le sens de:

[...] « Au milieu du xxe siècle les dommages du surpâturage étaient déjà évidents. Depuis, la capacité de charge par hectare a diminué de façon constante. Au tournant du xxi siècle, l'élevage ovin en Patagonie est devenu de moins en moins rentable, forçant à de nombreux éleveurs à augmenter la taille de leurs troupeaux sur des terres déjà fortement surpâturées.

La plus grande malédiction écologique de la Patagonie, cependant, peut être la source du salut de ses espaces naturels. Une grande partie de la Patagonie est encore inhabitée et ses champs de parcours s'étalent sur de vastes propriétés. Si l'on enlevait les moutons, les parcours auraient un répit et la chance de se régénérer. Une fois que les moutons et les clôtures seraient enlevés, les populations d'animaux sauvages restantes auront accès à l'habitat non fragmenté - et la chance de survivre et de prospérer »3. (Traduction des auteurs).

Sans aller jusqu'à prôner l'élimination des moutons, des idées intermédiaires se frayent un chemin dans la région. La proposition de parc naturel précédemment mentionnée pourrait cohabiter avec des zones ovines encore productives, tandis que d'autres seraient sacrifiées à des usages moins écologiques, tels que l'extraction minière ou pétrolifère, dans un aménagement du territoire qui devrait être entrepris à l'échelle régionale en Patagonie (Coronato \& al., 2011). Également, la reconversion à l'élevage des guanacos 
est une alternative qui s'amorce puisque semblerait compatible aux intérêts écologiques et économiques. Quant aux zones où l'élevage ovin demeure viable, elles ont aussi des propositions nouvelles de gestion du cheptel et du parcours afin d'assurer leur durabilité et d'augmenter leur productivité. Plusieurs initiatives non gouvernementales visant à un élevage ovin plus respectueux de l'environnement, apparaissent au travers d'organisations telles que l'OIA (Organización Internacional Agropecuaria) et OVIS 21, toutes deux bien insérées dans des réseaux internationaux partageant leurs buts, tels que l'IFOAM (International Federation of Organic Agriculture Movements) ou TNC (The Natural Conservancy) entre autres. Si ces initiatives privées réussissent à être en synergie avec les pouvoirs publics et leur action concernant l'élevage ovin, le glas de l'élevage ovin en Patagonie n’a pas encore sonné. Le dernier mot n’est peut-être pas encore dit.

\section{PROLANA}

« Programme d'assistance pour l'amélioration de la qualité de la laine ». L'aide de l'État aux producteurs ovins ne se borne pas aux subsides en cas d'aléas climatiques; il existe aussi une batterie de programmes d'appui de longue haleine, étant donné l'importance que les politiques publiques accordent à la filière ovine du point de vue sociale, en nombre d'emplois, et géopolitique, en termes d'occupation du territoire.

Même si le cycle de l'ovin qui façonna la Patagonie en tant que territoire et société est révolu et que le mouton bat en retraite, celui-ci demeure un repère identitaire majeur de la région. L'importance de cette identification est grande car la Patagonie, depuis ses origines, a été une région attirant des migrants très hétérogènes, ce qui rend difficile la construction d'un système collectif d'appartenance. Vraiment peu de repères identitaires sont communs à tous les Patagoniens. Le vent et la distance en seraient deux exemples, quoique négatifs. Il ne reste que le mouton qui rallie un peuplement étalé sur 15 degrés de latitude, des deux côtés de la cordillère des Andes (Coronato, 2010).

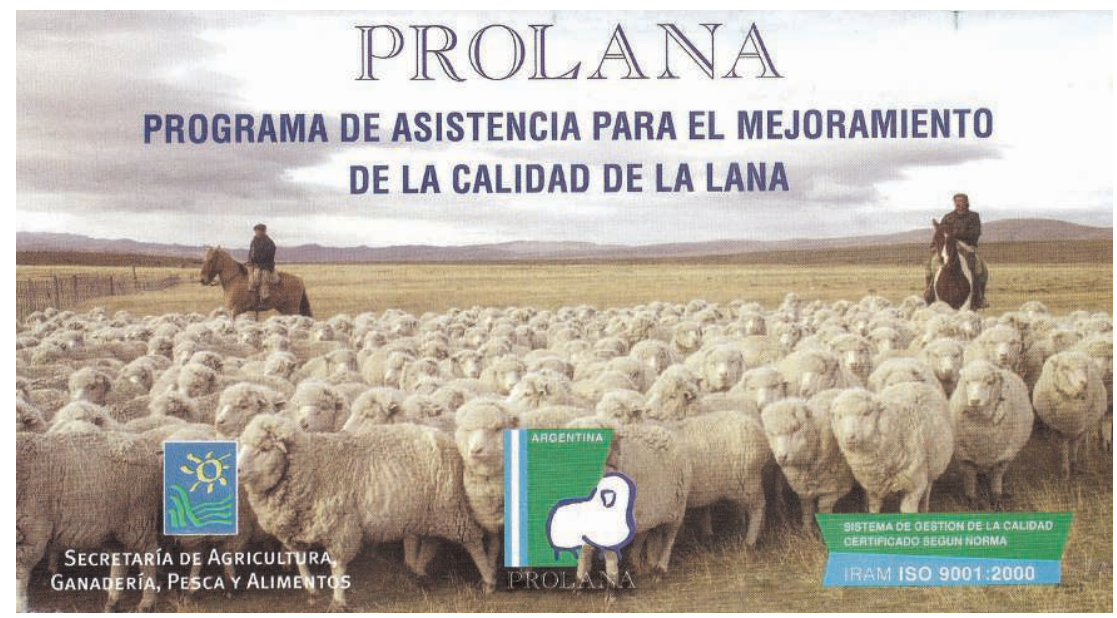

En admettant que « la Patagonie répond plutôt à une entité perceptive qu'à une structure territoriale et une certaine autonomie et cohérence fonctionnelle » (Bondel, 2004), on doit aussi admettre le rôle identitaire majeur du mouton dans la définition de la région. À présent le mouton contribue à bien moins de $5 \%$ du PBI régional et ne concerne directement qu'à une partie infime de la population. Toutefois, il demeure un symbole fortement ancré dans l'imaginaire collectif, du moment que « la force sociale de la représentation n'est pas nécessairement proportionnelle à sa valeur de vérité » (Bourdieu, 1980). Dans le cas patagonien ceci est vrai tant à l'extérieur qu'à l'intérieur de la région.

Pour l'extérieur, une seule image permet à elle seule de symboliser la Patagonie pour les étrangers à la région: les moutons. Une recherche conduite par Coronato (2010) montre que le mouton est le seul facteur identitaire accepté par tous comme représentatif pour l'ensemble de la région. Quelques autres facteurs dominent dans des régions particulières, cas les paysages grandioses, la faune marine et terrestre, les diverses cultures 


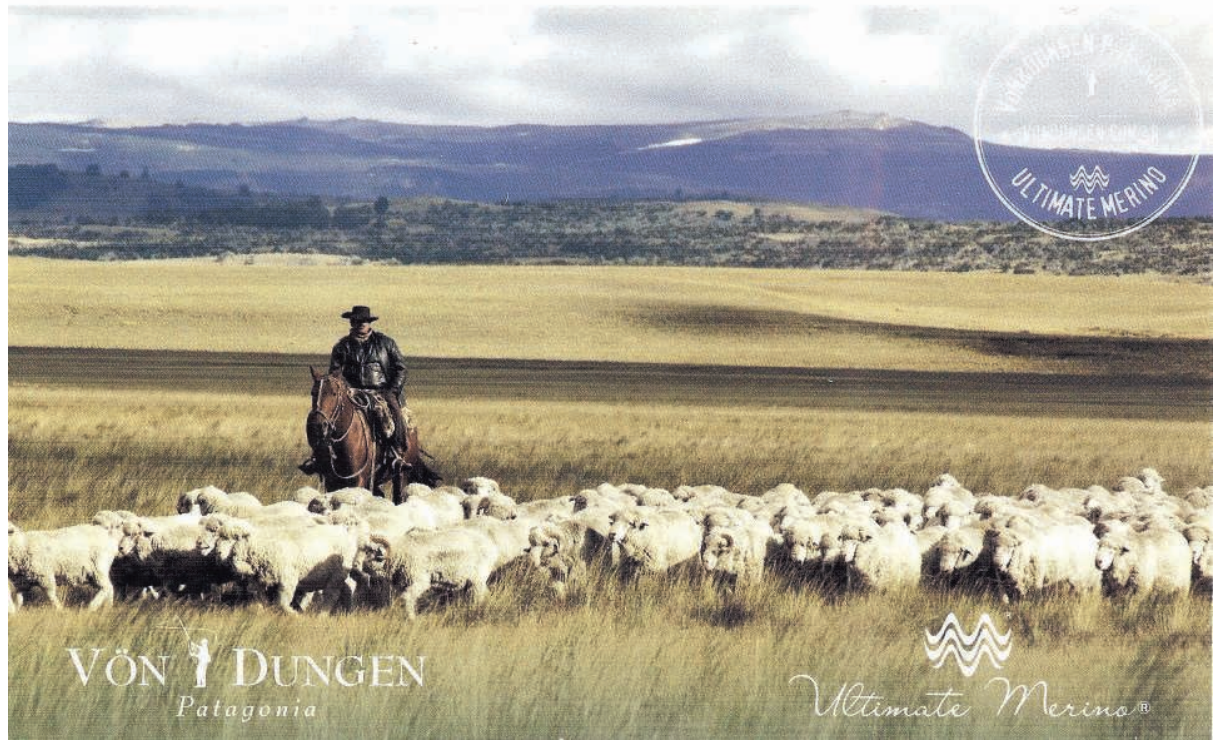

Ultimate Merino

La composition d'un troupeau de moutons, le gaucho à cheval (aux allures de cow-boy), l'herbe jaune et les montagnes au fond, forment le cachet identitaire parfait pour la publicité des laines de Patagonie. Le nom allemand et la marque en anglais sont aussi des indicateurs

d'une identité moutonnière très cosmopolite depuis le début de la colonisation.

\section{Exposition rurale}

Chaque été, des « expositions rurales» se tiennent dans les principales villes de Patagonie. Les animaux reproducteurs présentés par les différentes cabañas déploient leurs qualités dans un concours souvent serré et devant un jury exigeant, où des membres australiens ou néozélandais sont fréquents. Les animaux gagnants, les « champions », seront ensuite vendus aux enchères dans la foire même. Les « sociétés rurales » organisent ces expositions, qui ressemblent non seulement des éleveurs locaux mais de la Patagonie entière. En plus des enchères qui se tiennent lors de ces expositions, les grosses cabañas organisent leurs propres enchères quelques semaines plus tard. amérindiennes, les lacs... Un seul est présent partout, même s'il est rarement le premier: le mouton. Allant dans le même sens, dans la première géographie régionale argentine qui ne fut pas basée sur le milieu naturel (celle de Pierre Denis en 1920), la Patagonie y est au titre de « la région de lélevage de moutons », un peu comme s'il s'agissait de sa caractéristique majeure, ou comme s'il n'y avait pas de moutons ailleurs.

Dans l'imaginaire des Patagoniens eux-mêmes, on peut évaluer le lien entre le mouton et leur système d'appartenance au travers de deux manifestations symboliques facilement repérables: les monuments et l'héraldique. Les premiers peuvent être construits pour « fabriquer » un emblème ou pour en reconnaître son existence préalable. L'emblème est souvent l'outil des pouvoirs publics pour forcer une identité déterminée; en revanche, l'héraldique surgit spontanément au sein d'une communauté qui se reconnaît telle, ou qui veut exprimer sa reconnaissance à certaines valeurs. À côté des monuments nationalistes et religieux comme partout en Argentine et au Chili, la Patagonie compte de nombreux monuments, aussi simples que symboliques, témoignant la reconnaissance des communautés au mouton et à l'élevage ovin. L'homme n'est pas toujours présent dans la scène en tant que berger, mais l'association moutons $=$ pionniers est sous-entendue.

L'héraldique constitue aussi une autre source de la symbolique d'une communauté. Dans un pays neuf comme la Patagonie, l'héraldique n'est pas issue d'une obscure symbolique aux origines reculées, mais d'un choix conscient et recherché des emblèmes communautaires qui est fréquemment promu par les pouvoirs publics. Ceux-ci ont bien soin de consulter l'opinion de leurs administrés. Souvent, les emblèmes (armoiries et

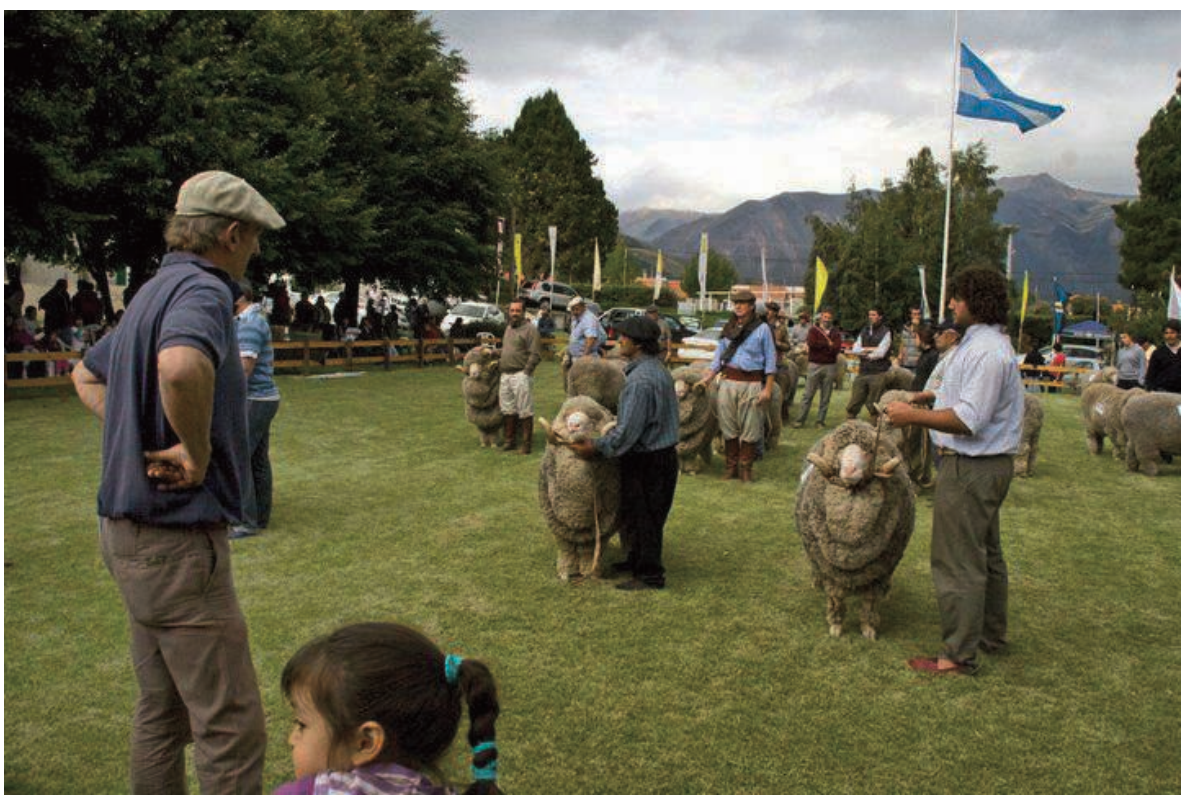


drapeaux) sont le fruit d'une sélection avec concours ouvert. Ainsi, les emblèmes présents dans l'héraldique de Patagonie sont de bons indicateurs des éléments représentant le système d'appartenance régionale. Excepté dans les îles Malouines, où un mouton domine les blasons, les ovins sont absents de toutes les armoiries des unités administratives de premier ordre, c'est-à-dire les provinces, mais en revanche, ils apparaissent dans l'héraldique de deuxième ordre, celle des municipalités. Ici, pas question du lion anglais, de l'hermine bretonne ou de l'aigle napoléonien; aucun symbole de force ou fierté, mais la simple reconnaissance de l'animal qui fit la richesse de la communauté et qui est un élément clé du patrimoine communautaire: le mouton. On les retrouve sur les armoiries de cinq des 18 communes du Chubut (28), deux de Santa Cruz (11 \%) et quatre du sud de Río Negro, sur le bord de la zone moutonnière. Plus au nord, en dehors des frontières politiques de la Patagonie, les moutons disparaissent complètement des armoiries des municipalités de La Pampa, où ils sont remplacés par des bovins, présents dans le $50 \%$ de cas. Nous sommes bien dans le domaine de la reconnaissance patrimoniale fondant à la fois l'identité du groupe et du territoire, tel que le décrit Bonerandi (2005).

On peut logiquement se demander pourquoi l'empreinte des moutons demeure si forte en Patagonie si le cycle ovin semble bel et bien fini et que l'activité ne contribue que si faiblement à l'économie régionale. Pourquoi ce symbole, qui est aussi le responsable indirect de la désertification de nombreux parcours et de l'anéantissement des peuples autochtones.

La réponse serait simple, et double à la fois. Premièrement, l'élevage ovin a été l'activité fondatrice de la communauté patagonienne; le mouton structura la société et son territoire. Chez tous les Patagoniens de souche il y a toujours un ancêtre qui gagna sa vie dans la filière ovine, éleveur, tondeur, berger, ouvrier dans la presse à balles ou employé dans les bureaux, peu importe, les aïeux sont toujours présents, d'autant plus que ce sont eux qui sont souvent à l'origine de la migration en Argentine. Deuxièmement, encore aujourd'hui et tout amoindri qu'il soit, l'élevage ovin la seule activité assurant l'occupation de l'ensemble de la région. S'il est vrai qu'à certains endroits, le mouton cohabite avec

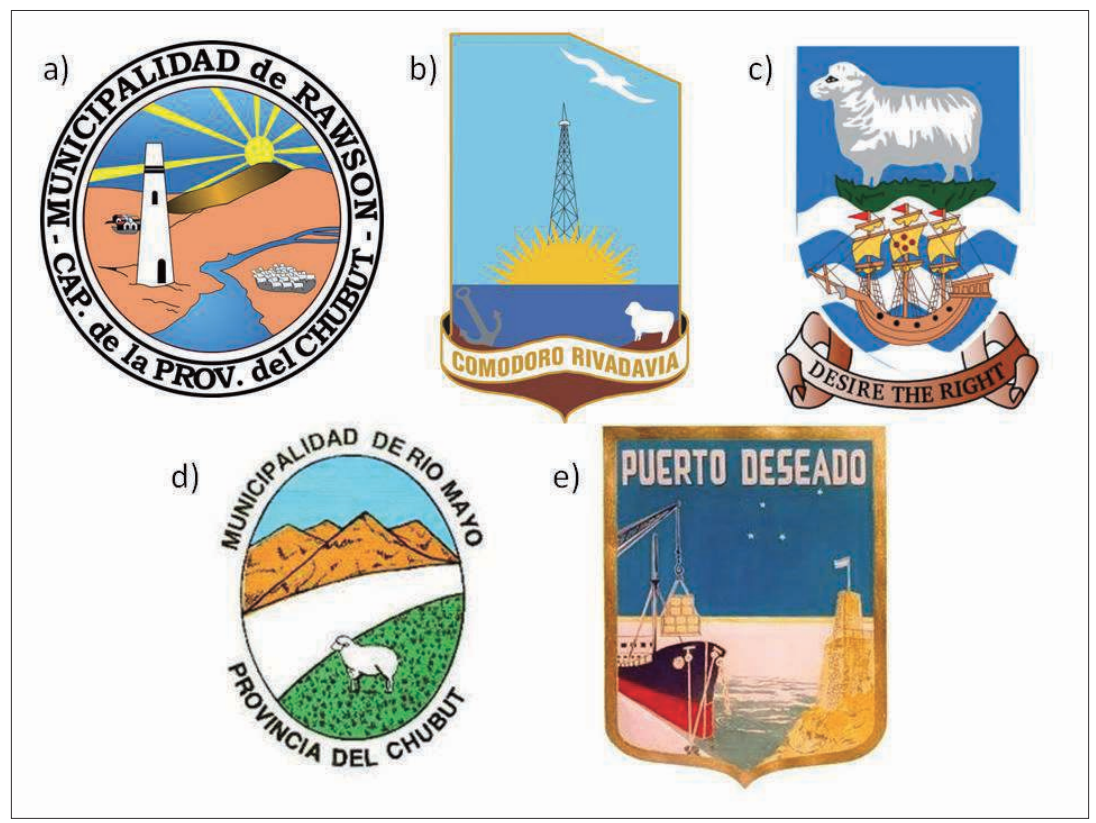

\section{Héraldique}

Le mouton est fréquent dans l'héraldique patagonne. Parmi d'autres exemples, on le retrouve ici dans les armoiries municipales de trois villes de la région (a, b, d) dont la préfecture de la province du Chubut (a) et la capitale économique régionale (b). Même absent, le mouton apparaît implicitement dans les balles que l'on exporte (e). II apparaît triomphant dans les armoiries des îles Malouines (c). À noter que dans tous les cas, le mouton pointe vers la gauche, l'ouest, ce qui pourrait impliquer la marche ancestrale de la colonisation ou l'habitude ovine d'avancer contre le vent. 
les puits de pétrole, ou apparîtt sur les cartes-postales de glaciers et lacs, il est aussi vrai que partout ailleurs en Patagonie, là où il n'y a pas de pétrole, ni de lacs ni de glaciers, il y aura certainement un mouton broutant la steppe désertifiée.

Pour finir, il nous semble que la réponse à la question du pourquoi encore s'identifier au mouton, est implicite dans le discours du président de la Société Rurale de Comodoro Rivadavia, vieux de vingt ans, mais toujours d'actualité ${ }^{4}$ :

« Parler de l'élevage ovin en Patagonie équivaut à parler de l'histoire même de cette terre. Il n'existe pas d'identité de cette région qui ne passe pas par le mouton; il n'existe pas non plus d'alternative agricole qui puisse modifier sérieusement cette réalité. Pendant beaucoup d'années la production ovine fut la base économique de la Patagonie et grâce à elle les fondements du peuplement et du développement furent établis. Le moutonnier affirma les frontières, conquit des espaces et fit naître d'innombrables localités de l'intérieur. Sa permanence et son activité ont joué un rôle géopolitique que nul ne peut dénier ».

Nul ne peut dénier non plus que le mouton que le Petit Prince demanda à Saint-Ex de dessiner était dans la caisse qu'il griffonna. Mais le pilote aurait bien pu griffonner une carte de la Patagonie et lui répondre: «Le mouton que tu veux est dedans ». Et çaurait été vrai.

\section{NOTES}

Photo d'ouverture: Le Fleuve. Les cours d'eau permanents sont peu nombreux dans les steppes de Patagonie. Les troupeaux qui en bénéficient sont des privilégiés, autant que leurs éleveurs (sur la photo, le fleuve Chubut à mi-chemin entre les Andes et l'Atlantique). Les fleuves traversent les plateaux dans des vallées abritées orientées Ouest-Est. La colonisation de la région a été dans le sens longitudinal et les fleuves, transversaux, ont joué le rôle d'étapes plutôt que de voie de pénétration.

Toutes les autres photos sont des auteurs.

1. Celle qui proposait « Colonizar a Amazônia pela pata do boi».

2. Discours de L. Etchevehere, président de la Sociedad Rural Argentina, août 2014. [www.edicionrural.com/0/nota/index.vnc?id=4469] (Consulté le 15 août 2014).
3. http://www.adventure-life.com/articles/patagoniabiodiversity-161/ (consulté le 9 janvier 2014).

4. Pablo Serres, discours prononcé lors de l'inauguration de l'exposition annuelle (n 55) en 1993.

\section{POUR CITER CET ARTICLE}

Coronato, F.-R. \& Tourrand, J.-F., 2015 Mouton, dessine-moi un pays. In Dupré, L., Lasseur, J. \& R. PoccardChapuis Pâturages Techniques \& Culture 63: 162-181 


\section{RÉFÉRENCES}

Andrade, L. 2005 Sociología de la desertificación. UNPA; Miño y Dávila: Buenos Aires.

Bernhardson, W. 1989 Land and life in the Falkland Islands (Islas Malvinas). University of California at Berkeley: Berkeley (Thèse de Doctorat en géographie).

Bonerandi, E. 2005 Le recours au patrimoine, modèle culturel pour le territoire? Géocarrefour 80 (2): 91-99.

Bondel, S. 2004 La Patagonia y el imaginario geográfico. Párrafos geográficos 3 :10. IGEOPAT. Trelew.

Bourdieu, P. 1980. L'identité et la représentation; éléments pour une réflexion critique sur l'idée de région. Actes de la Recherche en Sciences Sociales, 35: 63-70.

Coronato, F. 2010. Moutons et colons en Patagonie. Éditions Universitaires Européennes: Sarrebruck.

Coronato, F., Fasioli, E., Schweitzer, A., Tourrand, J.-F. 2011 Rethinking the role of sheep in local development of Patagonia, Argentina. 9 th International Rangeland Congress, Workshop: Livestock farming embedded in local development. Rosario : Argentina. https ://www.academia.edu/1645266/ Rethinking_the_Role_of_Sheep_in_Local_Development_of_Patagonia_Argentina. Consulté le 9 février 2015

Del Valle, H., Elissalde, N., Gagliardini, D. \& al. 1997 Desertification assess-ment and mapping in the arid and semi-arid regions of Patagonia (Argentina), Desertification Control Bulletin 31:6-11.

Defossé G., Robberecht R., 1987 Patagonia: Range management at the End of the World, Rangelands 9 (3) : 106-109.

Denis, P. 1987 [1920] La République Argentine. La mise en valeur du pays. Éditions del Solar: Buenos Aires.

Dobrée, P. 2006 El gran arreo. Zagier et Urruty: Ushuaia.

Lauenroth, W. 1998 Guanacos, spiny shrubs and the evolutionary history of grazing in the Patagonian steppe, Ecología Austral 8: 211-215.

Milchunas, D., Sala, O., Lauenroth, W. 1988 A Generalized model of the effects of grazing by large herbivores on grassland community structure, American Naturalist 132: 87-106.

Noy-Meir, I. 1995 Sustainability and management of natural resources in Patagonia. Proceedings of the International Workshop on Plant Genetic resources, Desertification and sustainability. Montes L et Oliva G, INTA: Rio Gallegos.

Obregón Iturra, J., Capdevila, L., Richard N. 2011 Les Indiens des frontières coloniales. Presses Universitaires de Rennes: Rennes.

Poccard-Chapuis, R. 2004 Les Réseaux de la conquête: filière bovine et structuration de l'espace sur les fronts pionniers d'Amazonie orientale brésilienne. Université de Paris X: Nanterre (Thèse doctorale en Géographie).

Roturier, S. \& Roué, M. 2015 Le Pâturage c'est toute une science! in Dupré, L., Lasseur, J. \& R. PoccardChapuis Pâturages, Techniques \& Culture 63: 92-109.

Scott, J, 2001 [1919] Libro del Centenario de San Julián: 1901-2001. Tomo 2. Municipalidad de San Julián: Santa Cruz.

Soriano, A. 2000 Andanzas de un ecólogo en la Patagonia. Sociedad Argentina de Botánica: Buenos Aires

Soriano, A., Paruelo, J. 1990 El Pastoreo ovino: principios ecológicos para el manejo de los campos, Ciencia Hoy 2 (7): 44-53.

Willis, B.1914 El Norte de la Patagonia: Naturaleza y riquezas. Ministerio de Obras Públicas: Buenos Aires. 


\section{RÉSUMÉ}

Mouton, dessine-moi un pays. L'élevage ovin et l'identité régionale en Patagonie. La Patagonie, extrémité australe des Amériques demeura terra incognita jusqu'à la fin du XIxe siècle, quand elle fut incorporée aux circuits politiques et économiques internationaux, et ceci grâce au développement de l'élevage ovin. La mise en valeur agricole de ces vastes steppes s'est faite à l'aide d'un élevage extensif qui s'est répandu en deux à trois décennies sur les quelques $800,000 \mathrm{~km} 2$ de la région. Le cheptel de Patagonie atteignit les vingt millions de moutons vers 1950, tandis que la population ne dépassait un million d'habitants qu'en 1980, de surcroît étant urbaine à 95 \%. Même si d'autres activités économiques, notamment l'exploitation pétrolière, se sont développées tôt et ont contribué à la formation d'une société très cosmopolite, le mouton est à la base de la société patagonienne, car cette dernière s'est structurée autour de la filière ovine. Malgré le déclin de l'activité dans les dernières décennies du xx à cause des crises économiques et environnementales, le caractère constitutif de l'élevage ovin dans la région explique la présence du mouton dans la symbolique régionale et sa persistance en tant que repère identitaire chez les Patagoniens.

\section{ABSTRACT}

Sheep, draw me a country! Sheep breeding and regional identity in Patagonia. Patagonia, the southernmost tip of the Americas, was terra incognita until the end of the 19 th century when it was incorporated into international political and economic circuits thanks to the development of sheep breeding. The agricultural enhancement of these vast steppes is the result of extensive breeding that spread over the region's some $800,000 \mathrm{~km} 2$ in just two to three decades. The Patagonian sheep herd numbered 20 million head by 1950, whereas the population of the region did not exceed one million inhabitants until 1980, $95 \%$ of them living in cities. Even if other economic activities, particularly oil drilling, developed early on and contributed to the formation of a highly cosmopolitan society, the sheep is at the core of Patagonian society, which is structured around the sheep sector. Despite the decline in activity in the last decades of the 20 th century because of economic and environmental crises, the constitutive nature of sheep breeding in the region explains the persistence of the sheep as a regional symbol and the fact that it is so closely linked to the identity of the Patagonian people.

\section{MOTS-CLÉS}

droit foncier, droit pastoral, pâture, patrimoine, territoire

\section{KEYWORDS}

sheep, pioneers, colonization, socio-ecological cycle 


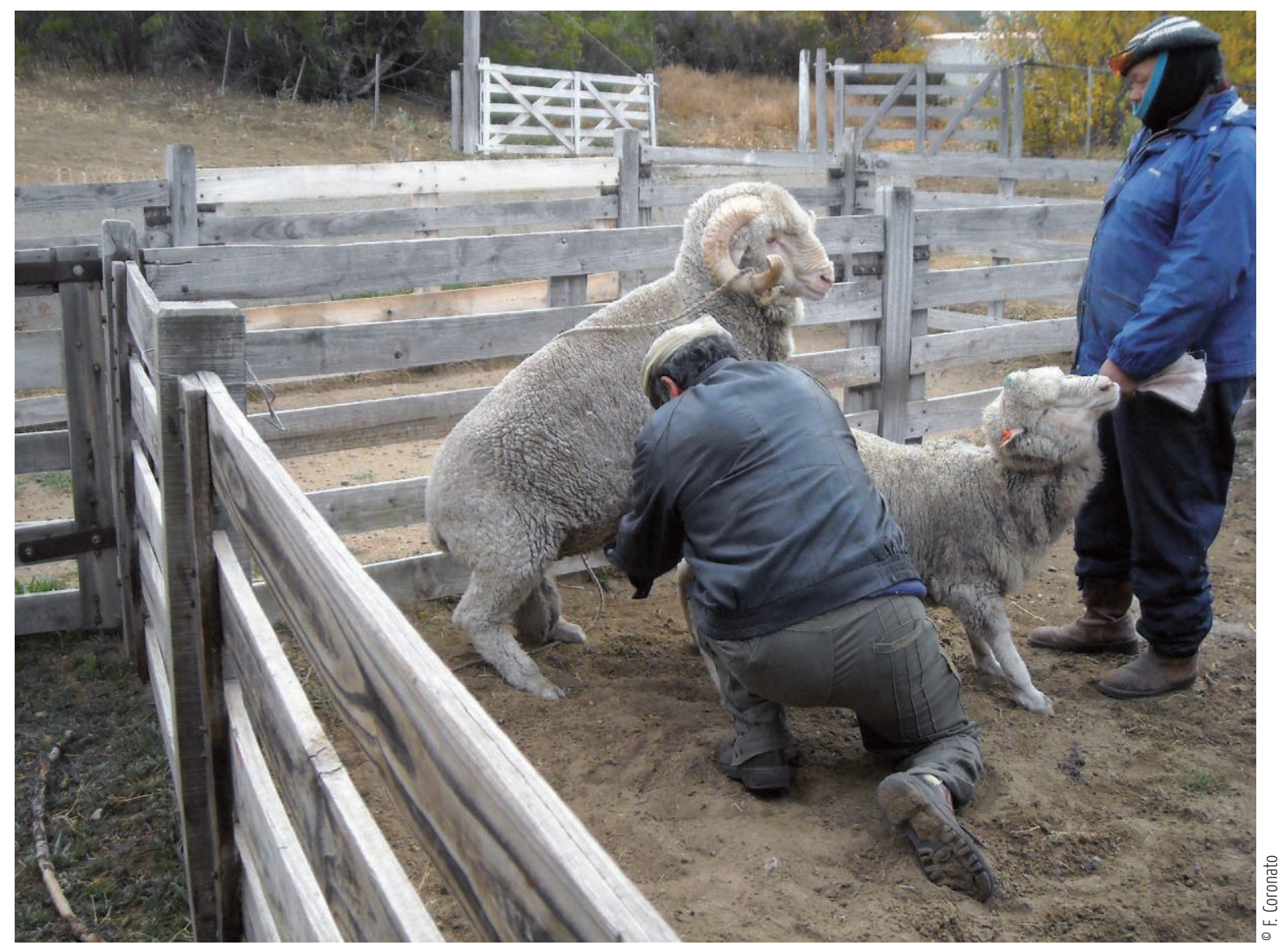

\section{Semen}

L'insémination artificielle n'est pas très répandue en Patagonie, mais les producteurs soucieux de la génétique la font de façon artisanale avec leurs propres reproducteurs (élevés ou achetés). Ici, le bélier est trompé par un vagin artificiel à l’aide une brebis fertile. La période des luttes s'étale de mars à mai, et du nord au sud de la région. 